\title{
Was bin ich? Qualitative Befunde zur Identifikation Türkeistämmiger in Deutschland
}

\section{Sascha Riedel}

In der öffentlichen und medialen Diskussion bildet die Identitätsbildung einen zentralen Streitpunkt der Integration von Migranten in Deutschland. Dies zeigt die immer wieder aufflammende Diskussion darüber, wann Migranten als Teil der Gesellschaft zu betrachten sind und wie viel Anpassung man von ihnen verlangen könne und/oder solle. Hier wird in jüngerer Vergangenheit neben dem empfundenen Heimatgefühl immer häufiger auch der muslimische Glaube diskutiert. ${ }^{1}$ Neben der Gruppengröße ist diese Tatsache ein ausschlaggebender Grund dafür, dass die Türkeistämmigen in der öffentlichen Diskussion um die Integration von Migranten dominieren. In der wissenschaftlichen Theoriebildung wird der Identitätsformation und deren Einfluss auf die Integration von Migranten hingegen weniger Aufmerksamkeit gewidmet. War die Identifikation in der klassischen Assimilationstheorie ${ }^{2}$ noch zentraler Bestandteil des Integrationsprozesses, wird in vielen neueren Modelle entweder keine separate Phase der "identifikativen Integration « definiert ${ }^{3}$ oder ihre Bedeutung als eher marginal ${ }^{4}$ angesehen. In quantitativen Studien wird die Identifikation mit Deutschland vielfach über wenige Items zur Selbstzuschreibung erfasst, ${ }^{5}$ obwohl eine Vielzahl englischsprachiger Literatur impliziert, dass eine Erfassung vielseitiger geschehen müsste. ${ }^{6}$

Der folgende Beitrag widmet sich daher der Frage, wie sich das Konzept »identifikativer Integration « sinnvoll eingrenzen und damit für die empirische Sozial-

\footnotetext{
1 Vgl. Frindte et al. 2011, Liljeberg 2012.

2 Vgl. Gordon 1964, Esser 1980.

3 Vgl. Alba, Nee 1997, Esser 2000, 2008.

4 Vgl. Haller et al. 2011, Portes, Zhou 1993.

5 Vgl. Constant, Zimmermann 2008, Frindte et al. 2011, Gesemann 2006, Haug et al. 2009, Liljeberg 2012.

6 Bourhis et al. 1997, Burnam et al. 1987, Hofstede et al. 1990, Knight und Kagan 1977, Phinney 1992, Phinney und Ong 2007, Singelis 1994; Suinn 2009, Unger et al. 2002, Watkins et al. 1997.
} 
forschung handhabbar machen lässt. Dafür werden Ergebnisse der von der Fritz Thyssen Stiftung finanzierten Studie "Phasen der Integration von Migranten « vorgestellt. Für diese wurden qualitative Leitfadeninterviews mit türkeistämmigen und russischsprachigen Migranten geführt. Vor allem aufgrund ihrer Schnittmenge zur muslimischen Gemeinde in Deutschland und ihrer Gruppengröße erscheinen die Türkeistämmigen für dieses Problem als besonders geeignete Analyseeinheiten. Der Aufbau des Beitrags gliedert sich wie folgt: Zuerst wird die theoretische Grundlage erarbeitet. Hierbei werden klassische und aktuelle soziologische und sozialpsychologische Theorien zur Identitätsbildung erläutert. Nach der theoretischen Einführung wird die Studie "Phasen der Integration « vorgestellt. Im Anschluss werden deren Ergebnisse diskutiert. Diese betreffen die Bezugspunkte und Dimensionen identifikativer Integration sowie die Kontexte, in denen die Ausgestaltung dieser stattfindet. Der Beitrag schließt mit offenen Fragen und einem Ausblick für zukünftige Forschung.

\section{Identität und Identifikation}

Die Diskussion der Identitätsbildung geht vielfach von einem übergeordneten »Selbst « aus, innerhalb dessen unterschiedliche Identitäten formiert werden. Das Selbst definiert Stryker "as constructed from diverse >parts.< [sic!] The parts which can be taken to comprise the self are discrete identities ${ }^{7}$. Vor diesem Hintergrund ist zu fragen, welche Teile das Selbst von Migranten definieren? Häufig wird in diesem Rahmen eine Gegenüberstellung von Aufnahmegesellschaft und ethnischer Herkunft vorgenommen. So argumentiert Gans, dass die Herkunft für Migranten häufig in einer symbolischen Ethnizität (symbolic ethnicity) ${ }^{8}$ Ausdruck findet. Diese stellt dabei vor allem eine Privatangelegenheit dar, die im Alltag der Personen von wenig Relevanz ist. Diese theoretische Position aktualisiert Waters, indem sie sagt, dass symbolische oder optionale Ethnizitäten in den USA nicht allen, sondern vor allem weißen, überwiegend europäischen Migranten zur Verfügung stehen. Schwarze und viele Migranten der Einwanderungsströme nach 1965 seien hingegen weniger frei, da ihre ethnische Zugehörigkeit unfreiwillig in einer Vielzahl von Kontexten (z. B. Schule, Arbeits- und Wohnungsmarkt) bedeutsam ist. Hierbei ist die Salienz der Identität wichtig. ${ }^{9}$ Diese bezeichnet die Wahrschein-

\footnotetext{
7 Stryker 1968, S. 559.

8 Symbolische Ethnizität bezeichnet eine überwiegend nostalgische und freizeitorientierte Bindung an die Herkunftsgesellschaft, die vor allem ab der dritten Generation von Einwanderern eintritt (Gans 1979, S. 6 ff.).

9 Vgl. Waters 2001.
} 
lichkeit, dass eine Identität über eine Vielzahl an Situationen oder über Personen hinweg in einer gegebenen Situation aktiviert wird..$^{10}$ Dabei ist es nach Waters' Argumentation für Personen verschiedener Herkunft unterschiedlich wahrscheinlich, dass ihre ethnische Abstammung in bestimmten Situationen bedeutsam wird.

Ein ähnlicher Ansatz findet sich in Modellen der situativen Ethnizität oder Identität. ${ }^{11}$ Die Identität eines Individuums bildet sich demnach nicht einmalig, sondern wird fortwährend situativ ausgestaltet. Dabei sind zwei Dimensionen für die Identitätsformation bedeutsam: die Rahmenbedingungen der sozialen Situation (strukturell) und die persönlichen Merkmale der beteiligten Individuen (kognitiv). Erstere bezieht Okamura hierbei vor allem auf soziostrukturelle Merkmale der Gruppen (z.B. Status, Bildung, Erwerbs- und Einkommensstruktur). Darüber hinaus sind die Beziehungen der verschiedenen Gruppen innerhalb der Gesellschaft und ihr relatives numerisches Verhältnis zueinander bedeutsam. Die kognitiven Merkmale beziehen sich hingegen darauf, welche subjektive Bedeutung die Ethnizität in der gegebenen Situation einnimmt und welche ethnische Kategorie dem Interaktionspartner zugeschrieben wird. ${ }^{12}$ In Einklang mit Waters' Argumentation ist auch in Ansätzen situativer Ethnizität die Identität nicht immer frei wählbar, da die strukturellen Bedingungen den Handlungsspielraum des Individuums maßgeblich beschränken können.

Die für den vorliegenden Beitrag zentrale Theorie stellt das Akkulturationsmodell von Berry dar. ${ }^{13}$ Den Begriff der Akkulturation definiert er dabei als "process that entails contact between two cultural groups, which results in numerous cultural changes in both parties « ${ }^{14}$. Die genannten kulturellen Änderungen bezieht er dabei auf die folgenden fünf Kategorien: physische (z. B. Wohnort und -bedingungen), biologische (z. B. Ernährung und Krankheiten), kulturelle (z.B. Sprache und Religion), soziale (z.B. Ingroup-Outgroup-Beziehungen) und psychologische Veränderungen (z. B. Verhalten und mentale Verfassung).$^{15}$ Die persönliche Migration (1. Generation), aber auch das Aufwachsen im Spannungsfeld zweier Kulturen (2. und nachfolgende Generationen) führt zu akkulturativem Stress, der das allgemeine Wohlbefinden und die Identitätsbildung der Personen beeinflusst. Dieser Stress ist umso geringer, je weniger Konfliktpotential es zwischen der Aufnahme- und Migrantengemeinde gibt. ${ }^{16}$

10 Vgl. Stryker und Burke 2000, S. 286.

11 Vgl. Okamura 1981, Alexander Jr., Wiley 1992.

12 Vgl. Okamura 1981, S. 453-455.

13 Vgl. Berry 1997, 2001.

14 Berry 2001, S. 616.

15 Vgl. Berry et al. 1987, S. 492.

16 Vgl. ebd. 
Im Folgenden werden alle Merkmale, die eine emotionale Bindung an Deutschland zur Folge haben, mit identifikativer Integration bezeichnet. Es ist jedoch auch möglich, dass Migranten ihre Herkunftsbindung im Aufnahmeland beibehalten. Daher argumentiert Berry, dass sich die Verbundenheit mit der Mehrheits- und Migrantengemeinde auf zwei voneinander unabhängigen Dimensionen ausgestaltet. Dabei sind vier Ausgänge der Akkulturation möglich: Assimilation meint eine überwiegende Orientierung an der Aufnahmegesellschaft, Segmentation hingegen eine ausschließliche Beibehaltung der eigenethnischen Bindung. Marginalisierte Migranten fühlen sich keiner der beiden Gemeinden; Personen mit einem Integrationsprofil sowohl der ethnischen als auch der Aufnahmegesellschaft zugehörig. ${ }^{17}$ Nach einem empirischen Test von Berry et al. weist mit 36,4 Prozent die Mehrheit der untersuchten Migranten das letztgenannte Muster auf. Die verbleibenden drei Akkulturationsprofile verteilen sich wie folgt: 22,5 Prozent (Segmentation), 22,4 Prozent (Marginalisierung) und 18,7 Prozent (Assimilation) ${ }^{18}$. Nach Bourhis et al. kann zudem noch der Typus des Individualisten unterschieden werden, den ein niedriges Zugehörigkeitsempfinden zur Herkunfts- und Aufnahmegesellschaft auszeichnet. Darüber hinaus messen Individualisten jedoch im Gegensatz zu marginalisierten Individuen Gruppenzugehörigkeiten allgemein wenig Bedeutung bei. ${ }^{19}$ Die genannten Profile lassen sich dann wie in Abbildung 1 anordnen.

Eine der wenigen gesamtheitlichen, deutschsprachigen Arbeiten zum Thema der Identifikation stellt Schnells »Dimensionen ethnischer Identität « dar. Er unterscheidet zwischen Kategorisierung, im Sinne einer persönlichen Klassifikation, und der stärker affektiven, ethnischen Identifikation. Letztere bezeichnet die individuelle Wertschätzung, die der entsprechenden Zugehörigkeit beigemessen wird. Diesen beiden Aspekten ordnet er spezifische Verhaltensweisen als dritte Dimension ethnischer Identität zu. ${ }^{20}$ Die Differenzierung zwischen einer kognitiven und einer affektiven Dimension findet sich auch in anderen Veröffentlichungen. ${ }^{21}$ Phinney und Ong benennen z. B. Handlungen, die einen Informations- oder Erfahrungsgewinn hinsichtlich der persönlichen Zugehörigkeit zur Folge haben,

17 Vgl. Berry 1997; Esser 2000.

18 Vgl. Berry et al. 2006, S. 313. Für diese Studie wurden 7997 Jugendliche zwischen 13 und 18 Jahren aus 13 Nationen, darunter u. a. Australien, Deutschland, Frankreich, Kanada und die USA, untersucht. Die Größe der untersuchten und nicht-repräsentativen Stichproben schwankt zwischen 240 und 1043 Befragten pro Nation. Neben Migranten wurden zudem auch »Einheimische « untersucht.

19 Vgl. Bourhis et al. 1997, S. 381.

20 Vgl. Schnell 1990, S. 45 f.

21 Vgl. Ashmore et al. 2004, Jasinskaja-Lahti, Liebkind 1999, Phinney, Ong 2007. 


\section{Abbildung 1}

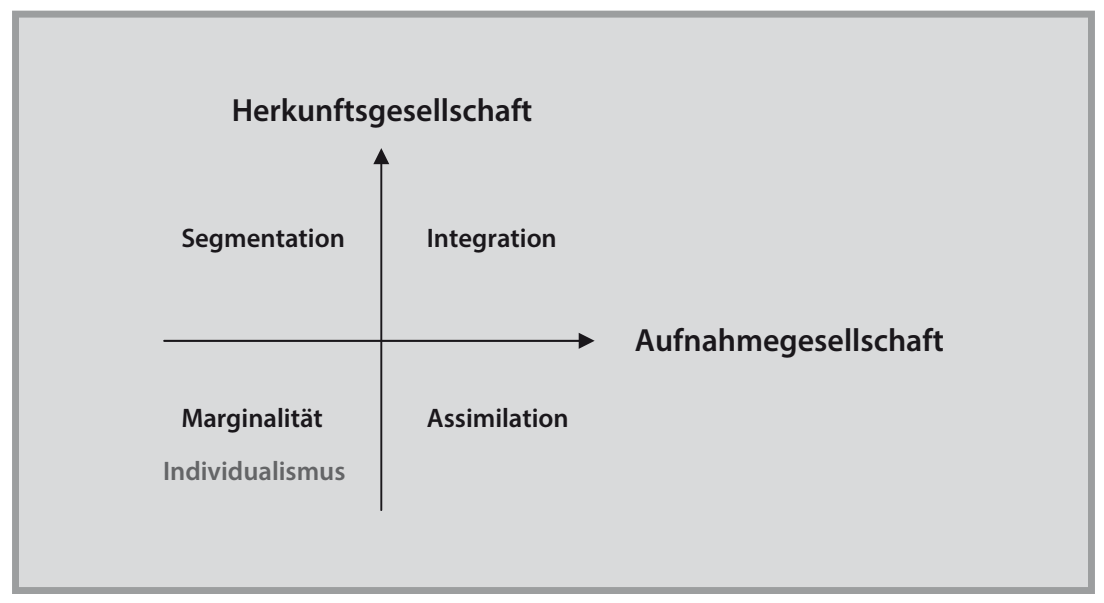

Tabelle 1 Identifikation im Überblick

\begin{tabular}{|c|c|c|}
\hline Ausgang & Orientierung & Items \\
\hline Assimilation & A & \multirow{4}{*}{$\begin{array}{l}\text { Sprachverhalten, Bräuche, Feiertage, religiöses Verhalten, } \\
\text { inter- und innerethnisches Kontakte, Selbstidentifikation } \\
\text { als Deutscher oder Ausländer, Gefühle der Heimatlosigkeit, } \\
\text { kulturelle Gewohnheiten (Musik, Video, Zeitungen, Ernäh- } \\
\text { rung), etc. }\end{array}$} \\
\hline Integration & $\mathrm{A} / \mathrm{H}$ & \\
\hline Marginalisierung & - & \\
\hline Separation & $\mathrm{H}$ & \\
\hline
\end{tabular}

A: Aufnahmegesellschaft, $\mathrm{H}$ : Herkunftsgesellschaft

mit exploration und den eher affektiven »sense of belonging « mit commitment. ${ }^{22}$ Tabelle 1 gibt einen Überblick über die Ausgänge der Identifikation und einige Beispielitems, wie sie Schnell vorgeschlagen hat. ${ }^{23}$

Im Folgenden wird die Studie »Phasen der Integration « vorgestellt, deren Ergebnisse im Anschluss mit Hinblick auf die dargestellten Theorien diskutiert werden.

22 Vgl. Phinney, Ong 2007, S. 272.

23 Vgl. Berry 1997, Esser 2000 bzw. Schnell 1990. 


\section{Die Studie: Phasen der Integration}

Bei der Studie wurden drei Ziele verfolgt: Erstens wurde die Identifikation von Migranten untersucht, wobei das Augenmerk auf den Bezugspunkten, Dimensionen und relevanten Kontexten der Migranten lag. Zweitens wurde versucht, besser geeignete Indikatoren für die Identifikation von Migranten zu entwickeln. Und drittens sollten die Phasen der Integration - wenn vorhanden - nachgezeichnet werden. Die Darstellung der Ergebnisse bezieht sich im Folgenden auf die beiden erstgenannten Punkte.

Um die genannten Ziele zu verwirklichen, wurden qualitative Leitfadeninterviews mit Türkeistämmigen ${ }^{24}$ und russischsprachigen Migranten geführt. Diese beiden Gruppen wurden dabei aufgrund ihrer Größe und Unterschiedlichkeit ausgewählt. Die Türken stellen mit zirka 2,49 Millionen Personen die größte Migrantengruppe Deutschlands ${ }^{25}$ und werden häufig bezüglich ihrer Integration als besonders problematisch wahrgenommen. Hinsichtlich Bildungserfolg ${ }^{26}$, Beschäftigungschancen ${ }^{27}$ und Spracherwerb ${ }^{28}$ weisen Studien für Deutschland auf die defizitäre Lage dieser Migrantengruppe hin. Im Gegensatz hierzu, scheint die Integration von Einwanderern aus der ehemaligen Sowjetunion unproblematischer und geradliniger zu verlaufen. ${ }^{29}$ Alle folgenden Angaben und Ergebnisse beziehen sich, sofern nicht anders angegeben, ausschließlich auf die untersuchten Türken.

\subsection{Methodik}

Als Erhebungsinstrument der Studie wurde die Methode des problemzentrierten Interviews gewählt. ${ }^{30}$ Als thematischer Schwerpunkt diente dabei die »Integration von Migranten in Deutschland « im Allgemeinen. Diese Problemstellung wurde den Teilnehmern als inhaltlicher Schwerpunkt kommuniziert und im Vorfeld unter theoretischen Gesichtspunkten analysiert. ${ }^{31}$ Eine spezielle Fokussierung auf die Identifikation mit Deutschland fand jedoch nicht statt.

Für die Leitfadenkonstruktion wurde ein biographischer Ansatz gewählt. Die Teilnehmer wurden gebeten, ihre Erfahrungen in Deutschland, beginnend mit

24 Aus Gründen der Lesbarkeit werden diese im Folgenden auch als Türken bezeichnet.

25 Vgl. Deutsches Statistisches Bundesamt 2011.

26 Vgl. Brettfeld, Wetzels 2007.

27 Vgl. Bender, Seifert 1996, Granato 2009, Kalter 2006.

28 Vgl. Haug et al. 2009, Kalter 2006.

29 Vgl. Haug 2005, Segeritz et al. 2010.

30 Vgl. Mayring 1993.

31 Vgl. ebd., S. 46. 
der Einreise oder der Kindheit darzustellen. Nachfragen bezogen sich im Anschluss auf den deutschen Spracherwerb, die Schulzeit, die Erwerbstätigkeit, den Freundeskreises und die Bindung an die Herkunfts- und Aufnahmegesellschaft. Um den Einfluss von Mitgliedern der Mehrheitsgesellschaft zu berücksichtigen, wurde ausführlich auf Erfahrungen der Teilnehmer eingegangen, in denen sie das Gefühl hatten mit der Fremdzuschreibung askriptiver Merkmale konfrontiert zu sein. Hierunter fällt z. B. ob und in welchen Situationen der Teilnehmer auf seine Herkunft angesprochen oder im Alltag mit seinem Migrationshintergrund konfrontiert wird.

Die transkribierten Interviews wurden nach einem subsumtionslogischen Verfahren mit dem Softwarepaket MAXQDA1o codiert, wobei die Dimensionen der klassischen Assimilationstheorie nach Esser und das Akkulturationsmodell von Berry als Grundlage dienten. Zusätzlich zur bereits genannten Quantifizierung der Transkripte wurde die Methodik der objektiven Hermeneutik angewandt, um das Datenmaterial auszuwerten. Diese erscheint im Rahmen des Ziels der Studie besonders geeignet, um »hinter den bewussten Entscheidungen liegende Regelmechanismen ${ }^{32}$ ausfindig zu machen. Da die identifikative Integration, wie an späterer Stelle noch diskutiert wird, zumindest partiell affektiv und unbewusst von Statten geht, würde eine ausschließliche Quantifizierung des Materials zu sehr eingeschränkte Ergebnisse liefern und der qualitativen Erhebungsmethode nicht gerecht werden.

\subsection{Deskriptive Statistiken}

Insgesamt wurden für die nicht-repräsentative Studie 39 Personen, darunter 24 Türkeistämmige, interviewt. Tabelle 2 zeigt die Staatsangehörigkeiten und Geburtsländer der türkischen Teilnehmer. Formal konnte zwischen erster und zweiter Generation ein Gleichgewicht verwirklicht werden. Allerdings sind zwei in Deutschland geborene Teilnehmer sogenannte Remigranten, die einige Jahre in der Türkei lebten und mit 16 und 18 Jahren erneut nach Deutschland eingewandert sind. Diese werden im Folgenden als Personen mit eigener Migrationsbiographie unter der ersten Generation gruppiert (Tabelle 3). Migranten, die vor ihrem zwölften Lebensjahr eingewandert sind und damit den Großteil der schulischen Laufbahn im Aufnahmeland absolviert haben, werden häufig als »1,5 generation « bezeichnet. ${ }^{33}$ Dieser Gruppe, der die Eingliederung annahmegemäß ge-

32 Brüsemeister 2000, S. 254.

33 Vgl. Rumbaut 1994, S. 759. 
Tabelle 2 Staatsangehörigkeit und Geburtsland

\begin{tabular}{lll}
\hline & Staatsangehörigkeit & Geburtsland \\
\hline Türkei & 13 & 12 \\
Deutschland & 12 & 12 \\
\hline
\end{tabular}

Tabelle 3 Deskriptive Statistiken

\begin{tabular}{|c|c|c|c|c|c|c|}
\hline & \multicolumn{2}{|c|}{ 1. Generation $(\mathrm{N}=14)$} & \multicolumn{2}{|c|}{ 2. Generation $(\mathrm{N}=10)$} & \multicolumn{2}{|c|}{ Summe } \\
\hline & $\varnothing$ & SE & $\varnothing$ & SE & $\varnothing$ & SE \\
\hline Geburtsjahr & 1966 & 2,4 & 1978 & 1,6 & 1971 & 1,9 \\
\hline Jahr der Migration & 1980 & 2,4 & - & - & - & - \\
\hline $\begin{array}{l}\text { Religiosität } \\
0=\text { gar nicht religiös } \\
4=\text { sehr religiös }\end{array}$ & 1,8 & 0,3 & 1,3 & 0,4 & 1,6 & 0,2 \\
\hline
\end{tabular}

genüber der ersten Generation leichter fallen sollte, gehören in der vorliegenden Stichprobe vier Personen an.

13 Personen haben die türkische, zwölf die deutsche und eine Person hat eine doppelte Staatsangehörigkeit. Das durchschnittliche Einreisealter der Personen mit eigener Migrationserfahrung liegt bei 14 Jahren (Tabelle 3$)^{34}$.

Wie aus Tabelle 3 deutlich wird, schätzen sich die Teilnehmer im Durchschnitt wenig religiös ein. Die Unterschiede zwischen den Generationen sind dabei als gering zu bewerten. Die Verteilung der Personen auf die unterschiedlichen Glaubensgemeinschaften ist in Tabelle 4 dargestellt.

Zwölf Teilnehmer fühlen sich dem Islam, sieben dem Alevitentum und vier Personen keiner Glaubensgemeinschaft zugehörig. Der verbleibende Befragte äußert, eine freie Glaubensrichtung zu haben.

34 Die durchschnittliche Interviewlänge lag bei zirka 27 Minuten. Sieben Teilnehmer haben das deutsche (Fach-)Abitur oder ein Studium abgeschlossen. Weitere sieben Personen haben einen Hauptschulabschluss. Die verbleibenden zehn Teilnehmer verteilen sich auf die Kategorien: Realschulabschluss, keinen Schulabschluss und türkische Schulabschlüsse exkl. Studium. 
Tabelle 4 Glaubensgemeinschaft

Tabelle 5 Geschlechterverteilung

\begin{tabular}{ll}
\hline muslimisch & 12 \\
alevitisch & 7 \\
keine & 4 \\
andere & 1 \\
\hline
\end{tabular}

\begin{tabular}{ll}
\hline männlich & weiblich \\
\hline 16 & 8 \\
\hline
\end{tabular}

Tabelle 5 zeigt die Geschlechterverteilung der Teilnehmer. Es wurden doppelt so viele männliche wie weibliche Teilnehmer befragt, was bei der Interpretation der Ergebnisse berücksichtigt werden muss.

\subsection{Ergebnisse}

Der Aufbau der Studie war explorativ angelegt, so dass a priori keine Hypothesen formuliert wurden. Aus den empirischen Daten der biographischen Interviews zeichneten sich jedoch Regelmäßigkeiten ab, die im Folgenden in Beziehung zu den theoretischen Konzepten gesetzt werden. Dabei werden zuerst die Bezugspunkte, danach die Dimensionen und abschließend relevante Situationen identifikativer Integration diskutiert. Diese allgemeine Gliederung wird im Folgenden anhand des konkreten Beispiels Türkeistämmiger in Deutschland illustriert.

\subsubsection{Bezugspunkte identifikativer Integration}

Nach wie vor ist die Frage, ob die Identitätsbildung von Migranten uni-, bi- oder multipolar erfolgt, unbeantwortet. ${ }^{35}$ Die Befunde der Studie »Phasen der Integration « weisen darauf hin, dass sich Türkeistämmige in Deutschland sowohl mit der Türkei, als auch mit Deutschland verbunden fühlen. Dies entspricht dem nach Berry zu erwartenden Regelfall identifikativer Integration. Die folgende Antwort auf die Frage, ob sich die Teilnehmerin eher deutsch oder türkisch fühlt, veranschaulicht diesen Fall:

»(...) natürlich manchmal überwiegt die deutsche Seite, manchmal die türkische (.) es kommt wahrscheinlich immer darauf an (,) auf (,) welcher Standpunkt gerade (...) akut ist (.)/also aktuell ist « (weiblich, geboren 1964, 1,5 Generation).

35 Vgl. Benet-Martínez, Haritatos 2005, Berry 1997, Esser 2000, Ryder et al. 2000. 
Mit 14 von 24 Teilnehmern äußerte die Mehrheit ein solches integratives Akkulturationsprofil. ${ }^{36}$ Die Angaben reichten dabei von »Deutsch-Türkin « über »halb halb « und "gespalten « bis hin zu der sehr präzisen Aussage sich zu »sechzig Prozent als Deutscher « zu fühlen. Drei der befragten Türkeistämmigen bekundeten hingegen, sich überwiegend als Deutsche zu fühlen. So äußerte folgender Teilnehmer:

»Deutschland (,) also ich sag mal so (,) das ist mein (,) das ist mein Vaterland « (männlich, geboren 1974, 2. Generation).

Diese Äußerung alleine würde nicht zwangsläufig für eine einseitige Bindung an Deutschland sprechen. Die drei genannten Personen äußern allerdings zusätzlich eine ausgeprägte Distanz zu ihrer »Herkunft «. So sagt z. B. der bereits zitierte Teilnehmer:

»Türkei ist für mich nur ein Urlaubsort (.) wenn ich Türkei sage (,) sehe ich nur (,) Strand, Meer, nichts Anderes (.) auch meine Familie da drüben (,) wir haben mit denen nichts zu tun « (männlich, geboren 1974, 2. Generation).

Vier Befragte fühlen sich überwiegend oder ausschließlich als Türken. Diese empfinden sich ausdrücklich als türkisch und nicht deutsch oder sehen eine persönliche Distanz zu Deutschland. Beispielhaft verdeutlicht folgendes Zitat ein solch nationales Profil:

$»(\ldots)$ ich fühl mich nicht Deutschen (.) tut mir Leid (...) also ich bin Türkin, bleib ich auch Türkin, deswegen habe ich also nicht deutschen Bürgerschaft also beantragt (...) ich will Türkin bleiben« (weiblich, geboren 1967, 1. Generation).

Die verbleibenden drei Personen äußern keine einzugrenzende, persönliche Verortung. Sie sagen zum Beispiel sich als »Kosmopolit « oder allgemein als » Mensch « zu fühlen und lehnen eine Festlegung auf Nationalstaaten ab. Dies entspricht demnach stärker dem Profil des Individualismus nach Bourhis et al. und weniger einer Marginalisierung, wie sie Berry ursprünglich formulierte. Ein Befragter äußerte z. B. auf die Frage, ob er sich eher als Türke oder eher als Deutscher fühlt:

»Trifft für mich nicht zu, ich habe keine nationale Gefühle (.) das ist was für Nationalisten und für Idioten « (männlich, geboren 1963, 1. Generation). 
Die Teilnehmer empfinden dabei sowohl eine Distanz zur Türkei als auch zu Deutschland. Diese geht jedoch über das Gefühl hinaus, sowohl in der »Heimat « als auch in Deutschland als Ausländer wahrgenommen zu werden, welches nahezu alle Befragten kennzeichnet. Zudem äußern Befragte mit einem individualistischen Profil häufig auch, nicht bloß eine Entscheidung zwischen der Herkunft und Deutschland treffen zu müssen. Sie wägen vielmehr zwischen einer Vielzahl an Optionen ab, wie das nachfolgende Zitat verdeutlicht:

»Türkei oder Deutschland (...) Deutschland oder Spanien, Deutschland oder Italien, Deutschland oder Brasilien (.) das sind die einzigen (,) Überlegungen (,) die ich jetzt für mich habe« (männlich, geboren 1976, 2. Generation).

Die in der Stichprobe bestehenden Identifikationsmuster weisen in dieselbe Richtung, wie die quantitativen Befunde von Berry et al. ${ }^{37}$ Der überwiegende Teil der 24 Befragten weist eine doppelte Bindung auf (58 Prozent). Zirka 17 Prozent lassen sich der Segmentation und 12,5 Prozent der Assimilation zuordnen. Im Gegensatz zu Berry erscheint es jedoch sinnvoll, die verbleibenden 12,5 Prozent nicht in einer diffusen Residualkategorie zu gruppieren, sondern diese als Individualisten und daher supranational orientiert zu klassifizieren. Dass unter günstigen Voraussetzungen auch Migranten erster Generation bereits eine starke Bindung an Deutschland entwickeln, verdeutlicht folgende Antwort auf die Frage, in welchen Bereichen sich der Interviewte als Teil der deutschen Gesellschaft fühlt:

»(...) ich kümmer meine Sohn und Töchter in Deutschland (.) das heißt (,) kann man nicht sagen fifty oder Halbes (,) ganz fühle ich doch (,) bin ich doch Deutschland Teil « (männlich, geboren 1977, 1. Generation).

Die gefundenen Identifikationsmuster sind zudem in Einklang mit neueren Theorien, die eine identifikative Integration auch für kleinräumige oder supranationale Einheiten vorsehen. ${ }^{38}$ Pries spricht in diesem Zusammenhang von segmentierten Identitäten, die sich aus lokalen, ethnischen, nationalen oder supranationalen Komponenten zusammensetzen. ${ }^{39}$ Das empirische Material unterstützt diese Überlegungen. Selbstzuschreibungen wie »Kölscher Türke« oder vergleichbare Aussagen, die eine lokale Bindung zum Wohnort Köln ausdrücken, wurden von fünf Teilnehmern genannt. Inwieweit jedoch nationale durch lokale Bezugs-

37 Vgl. Berry et al. 2006.

38 Vgl. Crul, Schneider 2010.

39 Vgl. Pries 1996, S. 469. 
punkte substituiert oder komplementiert werden können, müsste zukünftige Forschung untersuchen. Im folgenden Abschnitt wird nun dargestellt, welche Dimensionen identifikativer Integration sich eingrenzen lassen.

\subsubsection{Dimensionen identifikativer Integration}

Hinsichtlich der Dimensionen, in denen sich die Identifikation von Migranten ausdrückt, lassen sich aus den transkribierten Interviews in Einklang mit den zuvor erläuterten Theorien ${ }^{40}$ die folgenden zwei Dimensionen abstrahieren:

Erstens kann die Identität als Kategorisierung fungieren, wobei der Identität wenig emotionale Bedeutung zugewiesen wird. Dieser Aspekt soll im Folgenden, ähnlich wie die exploration bei Phinney und Ong die kognitive Dimension der Identität darstellen. Folgendes Beispiel verdeutlicht eine solche kognitive Identifikation:

»(...) man sitzt halt zwischen zwei Stühlen/das ist nun mal so (..) ich denke mal irgendwann (,) meine Enkelkinder, die werden sich als komplett Deutsche dann bezeichnen können (.) ich persönlich kann das nicht (.) ich bezeichne mich als Deutsch-Türkin « (weiblich, geboren 1986, 3. Generation).

Diese Befragte fordert, dass sich Türkeistämmige »den Gepflogenheiten « anpassen sollen, nennt sich selber eine »totale Gegnerin von Kopftüchern « und ist gegen den Bau von Moscheen in Deutschland. Zudem hat sie sich bewusst für die deutsche Staatsbürgerschaft entschieden und sagt zu dieser Entscheidung:

»(...) da habe ich mich auch selber gefragt (,) wie ich mich halt (,) wohin ich mich eher hingezogen fühle und (,) habe dann aber auch (,) relativ (,) schnell auch für mich klar entschieden (..) das ist meine Heimat, ich fühle mich hier wohl, dann will ich auch den deutschen Pass haben « (weiblich, geboren 1986, 3. Generation).

Darüber hinaus äußert sie:

»(...) auch wenn ich im Urlaub in der Türkei gefragt werde (...) wo meine Heimat ist, sage ich auch Deutschland, weil (..) Heimat ist ja da, wo man sich wohlfühlt halt « (weiblich, geboren 1986, 3. Generation).

Die türkische Herkunft dient dieser Teilnehmerin offensichtlich vor allem als Kategorie, der sie selber wenig emotionale Bedeutung beimisst. Diese kognitive

40 Vgl. Jasinskaja-Lahti, Liebkind 1999, Phinney, Ong 2007, Schnell 1990. 
Kategorisierung als Türkin, die im Alltag der Teilnehmerin wenig Relevanz hat und vorwiegend Freizeitaktivitäten, wie z.B. den Musikgeschmack betrifft, kann daher auch als symbolische Ethnizität bezeichnet werden: ${ }^{41}$

»zehn, zwanzig Prozent (,) meiner (,) Musik, die ich höre ist schon türkisch « (weiblich, geboren 1986, 3. Generation).

Häufig nennen die Teilnehmer - Sunniten und Aleviten gleichermaßen - in diesem Zusammenhang auch ihre Religion. Diese wird jedoch von allen Befragten, für die der Glaube eine Rolle spielt, als Privatsache betrachtet. Daher wird diese auch häufig in Zusammenhang mit weiteren Freizeitaktivitäten genannt, in denen sich ein Bezug zur Herkunft äußert:

»(..) also mein Glauben (,) ist typisch türkisch (...) also ich koche sehr gerne türkisch (.) ansonsten (,) ja das (,) wie das Kind also (,) Kinder erziehen und (,) halt solche Sachen « (weiblich, geboren 1974, 2. Generation).

Im Gegensatz hierzu zielt die zweite Dimension identifikativer Integration auf einen stärker affektiv ausgerichteten Aspekt ab. Die Annahme, die dieser affektiven Identifikation zu Grunde liegt, ist, dass z. B. die Aussage, »sich in Deutschland zu Hause zu fühlen ", auf latente Aspekte zurückzuführen ist. Problematisch könnte hierbei sein, dass diese den Individuen unter Umständen selber nur schwer zugänglich sind. Zur konsequenten Bewertung der identifikativen Integration von Migranten müsste zudem die »Mehrheitsgesellschaft « als Referenz ebenfalls untersucht und demnach vorab genau definiert werden. Dies kann im vorliegenden Beitrag jedoch nicht geleistet werden. Ein Versuch, die affektive Identifikation von Migranten zu erfassen, könnte die Frage nach der Internalisierung typischer Verhaltensweisen und Normen der Aufnahmegesellschaft darstellen. So erklären z. B. zehn Befragte, die stereotype deutsche Pünktlichkeit übernommen zu haben. Elf Teilnehmer fühlen sich, nach eigener Aussage, hinsichtlich ihrer Arbeitseinstellung und -moral im direkten Vergleich stärker deutsch als türkisch:

»(...) wenn eine Aufgabe da gemacht wird, dann hundert Prozent gemacht wird (.) und nicht zu fünfzig Prozent und dann alles andere naja (.) nein, hundert Prozent « (männlich, geboren 1964, 1. Generation)

Einen wesentlichen Einfluss hat in diesem Zusammenhang die gewählte Referenzkategorie - also ob sich die Teilnehmer mit Deutschen ohne Migrationshinter-

41 Vgl. Gans 1979. 
grund oder mit Personen aus der Türkei vergleichen. Ein Großteil der Befragten fühlt sich jedoch sowohl hinsichtlich der persönlichen Einstellungen, als auch in Bezug auf alltägliche Verhaltensweisen Deutschland näher als der Türkei. So äußert ein Teilnehmer:

»Von dem Tagesleben her (,) das ist ganz einfache Dinge, die man im Tagesleben hat (.) da merkt man `Da passt du nicht da rein $`$ (.) das ist ganz andere Realitäten da « [in der Türkei, d.V.] (männlich, geboren 1963, 1. Generation).

Ein anderer antwortet auf die Frage, ob er schon einmal darüber nachgedacht hat in die Türkei auszuwandern:

»Man kann da gut Urlaub machen, aber da leben (...) auch als Türke nicht (...) alleine schon wegen der Straße hier [in Deutschland, d. V.], die Ordnung mit den Autos und allem (.) sogar in einem Stau geht es geordnet zu (...) nein echt da leben [in der Türkei, d. V.] (,) nein « (männlich, geboren 1982, 2. Generation)

Im Alltag der Türkeistämmigen äußert sich die affektive Identifikation demnach nicht nur in allgemeinen Aussagen, wie z. B. sich in Deutschland zu Hause zu fühlen, sondern offenbart sich in konkreten Situationen. Wie dargestellt wurde, gestaltet sich dabei eine affektive Bindung unter anderem durch die Internalisierung deutscher Normen aus. Diese Übernahme relevanter Verhaltensweisen wird den Türkeistämmigen jedoch häufig erst in Kontrast zu Mitgliedern ihres Herkunftslandes bewusst:

»manchmal wenn ich nach Türkei (,) für die Urlaubszeit (,) dahin gehen (,) ich fühle mich wie eine Fremde da (.) das ist ganz anderes Menschen also (...) passe ich nicht dahin« (männlich, geboren 1965, 1. Generation).

Erst mit dem Wechsel des gewohnten Handlungskontextes werden vielen Migranten ihre internalisierten Verhaltensmuster bewusst. In Ermangelung besserer Ausdrucksmöglichkeiten, nennen viele Teilnehmer dann ihr »Gefühl«, um die affektive Identifikation auszudrücken. Allerdings ist dabei häufig, wie auch im folgenden Beispiel, die Internalisierung von Normen entscheidend, dass sich die Türkeistämmigen als Deutsche fühlen:

»(...) vom Gefühl her (,) fühle ich mich sowieso zu diesem Land (,) ich bin hier geboren, ich lebe hier (...) eigentlich habe ich nur noch Papier- (,) über Papierkram mit diesem Land [Türkei d. V.] zu tun« (männlich, geboren 1982, 2. Generation) 
So nennt der zitierte Befragte z. B. die Offenheit in Deutschland als persönliche Eigenschaft, die ihn von seinen »Landsleuten « abgrenzt:

»wir hier denken ja (,) man ist halt weltoffener hier (...) man hört einer Frau zu (..) hier kann ich (,) über Sachen reden, wo man (,) in anderen Ländern, das (,) überhaupt nicht geht (.) zum Beispiel« (männlich, geboren 1982, 2. Generation).

Die Bindung an Deutschland drückt sich dabei dadurch aus, dass die persönlichen Verhaltensweisen subjektiv stärker dem entsprechen, was der Teilnehmer als den deutschen und nicht den türkischen Regelfall empfindet. Auch hier ist allerdings eine doppelte Bindung möglich, da sich manche Verhaltensweisen an Deutschland und andere an der Türkei orientieren können. Zudem ist für Türkeistämmige ein großer Teil der internalisierten Normen sowohl mit dem Herkunfts- als auch mit der Aufnahmegesellschaft vereinbar.

Im Folgenden wird mit der Salienz ein Aspekt diskutiert, der bei der Identitätsformation in Deutschland insbesondere für Türkeistämmige von Bedeutung ist. Es werden zudem relevante Situationen vorgestellt, in denen die Salienz die identifikative Integration beeinflusst. Dies ist vor allem für die zukünftige empirische Sozialforschung von großer Bedeutung.

\subsubsection{Kontexte situativer Identitätsformation}

Erachtet man die vorgestellten Ansätze situativer Ethnizität auch für quantitative Untersuchungen als wichtig, müssten soziale Situationen ausgemacht werden, in denen die ethnische Herkunft formiert wird. In diesem Zusammenhang nimmt die Salienz der Ethnizität und damit die Wahrscheinlichkeit, dass die Herkunft in einer Vielzahl von Kontexten bedeutsam wird, eine zentrale Position ein. ${ }^{42}$ Dabei ist neben der tatsächlichen sozialen Distanz ${ }^{43}$ vor allem das empfundene Verhalten der Mehrheitsgesellschaft ein Prädiktor für das Ausmaß identifikativer Integration von Migranten. Wie sich sowohl im direkten Vergleich zu den russischsprachigen Studienteilnehmern, als auch in anderen Untersuchungen ${ }^{44}$ zeigt, sind Türkeistämmige in Deutschland stärker als die meisten anderen Migrantengruppen mit sozialer Distanz konfrontiert. Dies ist ein weiterer Grund, dass sich diese für die Diskussion der identifikativen Integration besonders eignen. Für die Erfassung diskriminierender Handlungen erscheint nach Feagin eine Gliederung

42 Vgl. Stryker, Burke 2000.

43 Vgl. Bogardus 1951, Parrillo, Donoghue 2005.

44 Vgl. Böltken 2000. 
in unterschiedliche Formen der Diskriminierung sinnvoll. Die Bandbreite reicht hierbei vom Wechseln der Straßenseite über benachteiligende Handlungen bis zur Androhung und Anwendung von Gewalt. ${ }^{45}$ Dabei liegt der Fokus im Folgenden auf weniger offensichtlichen Handlungen, weswegen offene verbale oder körperliche Attacken, sowie deren Auswirkungen außen vor gelassen werden. Angesichts der vorliegenden Daten erscheinen zumindest drei Kontexte relevant, in denen Handlungen der Mehrheitsmitglieder die Ausgestaltung der identifikativen Integration von Migranten beeinflussen. Diese Liste beansprucht jedoch keine Vollständigkeit.

An erster Stelle kann an den Alltag, wie z. B. Einkäufe oder die Erwerbstätigkeit gedacht werden. Beispielhaft hierfür lässt sich folgende Aussage zitieren:

»Also es waren bei meiner Klasse schon Kinder (,) Mädchen, die mit Kopftuch gekommen sind und das war schon ein Problem für den (...) bei den meistens Kinder, bei den deutschen Kinder hat das gestört irgendwie (,) ich weiß nicht warum « (weiblich, geboren 1971, 1,5 Generation).

Zweitens können institutionelle Akteure eine empfundene Benachteiligung verursachen. Schulen und Behörden erscheinen hier besonders relevant. Folgende Antwort auf die Frage, in welcher Situation die Teilnehmerin das Gefühl hatte, sich zwischen der Türkei und Deutschland entscheiden zu müssen, veranschaulicht dies:

»(..) wenn man lange arbeitslos ist (,) ist natürlich schwer (...) also Behörden also Hartz 4 (...) Jobcenter (.) die dich also letzte Dreck behandelt « (weiblich, geboren 1967, 1. Generation).

Drittens ist auch die öffentliche Wahrnehmung, neben der Berichterstattung von Presse und Medien, vor allem in Form von Äußerungen politischer Eliten, bedeutend für die Identifikation von Migranten. Auch hier zeigen die empirischen Ergebnisse, dass sich die Türkeistämmigen mit den größten Hindernissen konfrontiert sehen. Dies wird deutlich, wenn selbst folgende Teilnehmerin, die von sich selber sagt, »laizistisch aufgewachsen « zu sein, in Bezug auf die Integrationsdebatte in Deutschland meint:

»(...) die Debatte ist total schief (,) also finde ich (.) ist ja keine Integrationsdebatte mehr/das ist ja schon eine Islamdebatte finde ich (.) das geht ja immer nur um Moslems (...) bei Hart aber Fair oder bei Maischberger sind immer irgendwelche Türken 
und es wird immer über Türken diskutiert, wenn es um die Integration geht « (weiblich, geboren 1981, 2. Generation).

Trotz einer offen vertretenen Distanz, sowohl zum türkischen Staat als auch zum Islam, empfindet die Befragte das Thema der Integration und den öffentlichen Diskurs um dieses als störend. Wie die dargestellten Zitate verdeutlichen, ist die Salienz der ethnischen Herkunft für Türkeistämmige in Deutschland sehr hoch. Aus diesem Grund findet sich auch nur für wenige Teilnehmer eine ausschließlich symbolische oder optionale Ethnizität. ${ }^{46}$ Für die meisten spielt ihre Herkunft auch im Alltag auf die eine oder andere Weise eine Rolle und behindert damit sowohl die kognitive als auch die affektive Identifikation mit Deutschland.

\section{Diskussion}

Die dargestellte Studie dient dem Zweck, die Identifikation Türkeistämmiger in und mit Deutschland zu beleuchten. Dabei muss beachtet werden, dass die kleine Fallzahl von 24 Teilnehmern keine inferenzstatistischen Schlüsse zulässt und die Befunde somit lediglich die Grundlage zukünftiger Forschung bilden können. Die Ergebnisse zeigen, dass Türkeistämmige in Deutschland mit Hindernissen konfrontiert werden, die ihre identifikative Integration behindern. Die Salienz ihrer ethnischen Herkunft beeinflusst sowohl die kognitive, als auch die affektive Identifikation. Dennoch äußern die meisten Befragten sich in ihrem Verhalten Deutschland angenähert zu haben. Ob dies tatsächlich der Fall ist oder lediglich so empfunden wird, lässt sich nicht nachvollziehen, da die Einschätzungen über das individuelle Verhalten auf Vermutungen der Befragungsteilnehmer beruhen. Die Grundlage bilden dabei die Stereotype der interviewten Personen über Deutsche ohne Migrationshintergrund. Für eine Bewertung der tatsächlichen Entwicklung müssten als Referenzkategorie auch Personen ohne Migrationshintergrund untersucht werden. Besonders exponiert empfinden die Teilnehmer dabei die Unterschiede ihres Verhaltens im Kontrast zu Personen, die in der Türkei leben.

Für die kognitive Identifikation und damit die Kategorisierung ihrer Herkunft, zeigt sich ein ganz ähnliches Bild. Bei der Einschätzung, ob sich Türkeistämmige als Deutsche betrachten, reflektieren sie neben ihrer eigenen Einschätzung auch die Salienz ihrer ethnischen Herkunft. Die Befunde der Studie weisen in Einklang mit den dargestellten US-amerikanischen Studien darauf hin, dass sich die meisten Türkeistämmigen in Deutschland sowohl als Türken als auch als Deutsche ka-

Vgl. Gans 1979, Waters 2001. 
tegorisieren ${ }^{47}$ Darüber hinaus können auch supranationale Einheiten, wie Europa, oder lokale Bezugspunkte, wie der Wohnort, Grundlage einer identifikativen Integration bilden.

Es zeigt sich jedoch im Gegenzug auch, dass bei der kognitiven, anders als bei der affektiven Identifikation, eine vollständige Abwendung von Deutschland möglich ist. Während die Übernahme gewisser Normen und Verhaltensweisen unumgänglich erscheint und lediglich in der Intensität variiert, ist es durchaus möglich sich rein kognitiv vom Aufnahmeland abzuwenden. Hier zeigen sich die für Türkeistämmige in Deutschland besonders ausgeprägten, wechselseitigen Interdependenzen zwischen den drei zentralen Aspekten des Beitrags: Salienz, kognitive und affektive Identifikation. Die Interviews zeigen, dass eine empfundene Distanz zu Mitgliedern der Mehrheitsgesellschaft in bestimmten Situationen Einfluss auf die Identifikation nimmt. Dabei kann neben der kognitiven auf lange Sicht auch die affektive Identifikation eingeschränkt werden, wenn es zu einem bewussten Rückzug in ethnische Enklaven kommt. Diese Wechselwirkung zwischen Fremdund Eigenwahrnehmung lässt sich in Deutschland für die Türkeistämmigen besonders deutlich nachzeichnen. Die qualitativen Ergebnisse implizieren daher, dass die genannten Aspekte auch für quantitative Studien berücksichtigt werden müssen, um valide Aussagen über die Identifikation Türkeistämmiger in Deutschland treffen zu können. Hieraus ergeben sich Probleme, die im Folgenden diskutiert werden.

\section{Ausblick}

Die dargestellten Befunde weisen darauf hin, dass die Identifikation von Migranten allgemein und von Türken im speziellen, ein mehrdimensionaler und wechselseitig beeinflusster Prozess ist. Dies führt zu einem zentralen Problem heutiger Sozialforschung auf diesem Gebiet: Für die Identifikation von Migranten stehen keine zufriedenstellenden quantitativen Messinstrumente zur Verfügung. In der Regel arbeiten Studien, wie z. B. das Sozioökonomische Panel (SOEP), mit Items, mit denen ausschließlich danach gefragt werden kann, wie sehr sich jemand als Deutscher und/oder Ausländer fühlt. Hiermit werden jedoch die drei zentralen Aspekte - die kognitive und affektive Identifikation sowie die Salienz der Herkunft - konfundiert gemessen. ${ }^{48}$ Zudem beschränkt sich die Messung der Salienz

47 Vgl. Berry 1997, 2001, Berry et al. 2006.

48 Das SOEP erfasst die Identifikation z. B. mit den beiden Items: 1) Wie sehr fühlen Sie sich als Deutscher? 2) Und wie sehr fühlen Sie sich noch dem Land verbunden, aus dem Sie oder Ihre Familie kommen? 
der ethnischen Herkunft bislang in der Regel auf offene Diskriminierungen. So erfasst z. B. das SOEP diesen Aspekt über das folgende Item zur empfundenen Benachteiligung: Wie häufig haben Sie persönlich innerhalb der letzten beiden Jahren die Erfahrung gemacht, hier in Deutschland aufgrund Ihrer Herkunft benachteiligt worden zu sein?

Unter Berücksichtigung der dargestellten Befunde sollten jedoch unterschiedliche situative Kontexte und Handlungen unterschieden werden. Es erscheint daher für zukünftige Forschung wichtig, die genannten Dimensionen der identifikativen Integration getrennt und vor allem trennscharf zu erfassen.

Eine weitere verbleibende Frage für zukünftige Forschung ist zudem, wie sich die identifikative Integration für die dritte und nachfolgende Generationen ausgestaltet. Eine Befragte äußerte z. B. auf die Frage, ob sie sich eher türkisch oder deutsch fühlt:

»Das können meine Kinder glaube ich in zehn Jahren beantworten/die würden dann sagen $>$ Wir sind Deutsche « (weiblich, geboren 1981, 2. Generation).

Zukünftige Forschung hätte demnach drei Aufgaben zu erfüllen. In einem ersten Schritt müsste ein geeignetes Messinstrument zur quantitativen, differenzierten und vollständigen Erhebung der identifikativen Integration erarbeitet werden. Hier sollte vor allem die Kontext- und Situationsabhängigkeit, die für die Salienz der Ethnizität bedeutsam ist, berücksichtigt werden. Zweitens wären die entwickelten Items, idealerweise repräsentativ, zu erheben und es müsste drittens erörtert werden, in welchem Verhältnis die identifikative Integration zur sprachlichen, strukturellen und sozialen Integration steht. Hier wäre z. B. nach der Theorie der segmentierten Assimilation zu erwarten, dass sich für verschiedene Migrantengemeinden unterschiedliche Muster der Identifikation und Integration zeigen.$^{49}$ Dabei stellen die Türkeistämmigen auch für zukünftige Studien eine besonders interessante Untersuchungsgruppe dar, da diese in Deutschland stärker als andere Migranten mit sozialer Distanz konfrontiert sind. ${ }^{50}$ Vor allem die Wechselwirkung zwischen Mehrheits- und Minderheitsgesellschaft sollte sich mit einem überarbeiteten Messinstrument genauer nachzeichnen lassen. In Anbetracht der anhaltenden medialen Präsenz und Emotionalität des Themas, erscheint dies als lohnendes Ziel zukünftiger Forschung.

49 Vgl. Haller et al. 2011, Portes, Zhou 1993.

50 Vgl. Böltken 2000. 


\section{Quellen- und Literaturverzeichnis}

Alba, R., Nee, V. 1997. Rethinking Assimilation Theory for a New Era of Immigration. International Migration Review 31 (4): 826-874.

Alexander Jr., C. N., Wiley, M. G. 1992. Situated Activity and Identity Formation. In Social psychology: Sociological perspectives, hrsg. M. Rosenberg, R. H. Turner, 269-289. New Brunswick: Transaction.

Benet-Martínez, V., Haritatos, J. 2005. Bicultural identity integration (BII): Components and psychosocial antecedents. Journal of Personality 73 (4): 1015-1050.

Berry, J. W. 1997. Immigration, Acculturation, and Adaptation. Applied Psychology: An International Review 46 (1): 5-34.

Berry, J. W. 2001. A Psychology of Immigration. Journal of Social Issues 57 (3): 615-631.

Berry, J. W., Kim, U., Minde, T., Mok, D. 1987. Comparative Studies of Acculturative Stress. International Migration Review 21 (3): 491-511.

Berry, J. W., Phinney, J.S., Sam, D. L., Vedder, P. 2006. Immigrant Youth: Acculturation, Identity, and Adaption. Applied Psychology: An International Review 55 (3): 303-332.

Bender, S., Seifert, W. 1996. Zuwanderer auf dem Arbeitsmarkt: Nationalitäten- und geschlechtsspezifische Unterschiede. Zeitschrift für Soziologie 25 (6): 473-495.

Böltken, F. 200o. Soziale Distanz und räumliche Nähe: Einstellungen und Erfahrungen im alltäglichen Zusammenleben von Ausländern und Deutschen im Wohngebiet. In Deutsche und Ausländer: Freunde, Fremde oder Feinde? Empirische Befunde und theoretische Erklärungen, hrsg. R. Alba, P. Schmidt, M. Wasmer, 147-194. Wiesbaden: Westdeutscher Verlag.

Bogardus, E. S. 1951. Measuring Changes in Ethnic Reactions. American Sociological Review $16(1): 48-51$.

Bourhis, R. Y., Moise, L. C., Perreault, S., Senecal, S. 1997. Towards an Interactive Acculturation Model: A Social Psychological Approach. International Journal of Psychology 32 (6): $369-386$.

Brettfeld, K., Wetzels, P. 2007. Muslime in Deutschland - Integration, Integrationsbarrieren, Religion sowie Einstellungen zu Demokratie, Rechtsstaat und politisch-religiös motivierter Gewalt. Ergebnisse von Befragungen im Rahmen einer multizentrischen Studie in städtischen Lebensräumen, hrsg. vom Bundesministerium des Innern. Berlin.

Brüsemeister, T. 2000. Qualitative Forschung: Ein Überblick. Wiesbaden: Westdeutscher Verlag.

Burnam, M. A., Telles, C. A., Karno, M., Hough, R. L., Escobar, J. I. 1987. Measurement of Acculturation in a Community Population of Mexican Americans. Hispanic Journal of Behavioral Sciences 9 (2): 105-130.

Constant, A. F., Zimmermann, K. F. 2008. Integration von Migranten. Ethnische Identität bestimmt ökonomischen Erfolg. Wochenbericht des DIW Berlin 42: 644-650. http:// www.diw.de/documents/publikationen/73/diw_01.c.89605.de/o8-42-1.pdf. Zugegriffen: 5. Dezember 2012.

Crul, M., Schneider, J. 2010. Comparative integration context theory: participation and belonging in new diverse European cities. Ethnic and Racial Studies 33 (7): 1249-1268.

Deutsches Statistisches Bundesamt 2011. Bevölkerung und Erwerbstätigkeit: Bevölkerung mit Migrationshintergrund - Ergebnisse des Mikrozensus 2010. Wiesbaden. 
Diehl, C., Schnell, R. 2006. >Reactive Ethnicity` or >Assimilation «? Statements, Arguments, and First Empirical Evidence for Labor Migrants in Germany. International Migration Review 40 (4): 786-816.

Esser, H. 1980. Aspekte der Wanderungssoziologie. Assimilation und Integration von Wanderern, ethnischen Gruppen und Minderheiten. Eine handlungstheoretische Analyse. Darmstadt: Luchterhand.

Esser, H. 2000. Die Konstruktion der Gesellschaft. Frankfurt/Main: Campus.

Esser, H. 2008. Assimilation, ethnische Schichtung oder selektive Akkulturation? Neuere Theorien der Eingliederung von Migranten und das Modell der intergenerationalen Integration. In Migration und Integration, hrsg. F. Kalter, 81-107. Wiesbaden: VS.

Esser, H., Friedrichs, J. (Hrsg.) 1990. Generation und Identität: Theoretische und empirische Beiträge zur Migrationssoziologie. Opladen: Westdeutscher Verlag.

Feagin, J.R. 1991. »The Continuing Significance of Race: Antiblack Discrimination in Public Places. American Sociological Review 56 (1): 101-116.

Frindte, W., Boehnke, K., Kreikenbom, H., Wagner, W. 2011. Lebenswelten junger Muslime in Deutschland. Ein sozial- und medienwissenschaftliches System zur Analyse, Bewertung und Prävention islamistischer Radikalisierungsprozesse junger Menschen in Deutschland, hrsg. vom Bundesministerium des Innern. Berlin.

Gans, H. J. 1979. Symbolic ethnicity: the future of ethnic groups and cultures in America. Ethnic and Racial Studies 2 (1): 1-20.

Gesemann, F. 2006. Die Integration junger Muslime in Deutschland. Bildung und Ausbildung als Schlüsselbereiche sozialer Integration. Berlin: Politische Akademie der FriedrichEbert-Stiftung.

Granato, N. 2009. Effekte der Gruppengröße auf die Arbeitsmarktintegration von Migranten. Kölner Zeitschrift für Soziologie und Sozialpsychologie 61: 387-409.

Haller, W., Portes, A., Lynch, S. M. 2011. Dreams Fulfilled, Dreams Shattered. Determinants of Segmented Assimilation in the Second Generation. Social Forces 89: 733-762.

Haug, S. 2005. Zum Verlauf des Zweitspracherwerbs im Migrationskontext: Eine Analyse der Ausländer, Aussiedler und Zuwanderer im Sozio-ökonomischen Panel. Zeitschrift für Erziehungswissenschaft 8 (2): 263-284.

Haug, S., Müssig, S., Stichs, A. 2009. Muslimisches Leben in Deutschland. Forschungsbericht im Auftrag der Deutschen Islam Konferenz 6, hrsg. vom Bundesamt für Migration und Flüchtlinge. Nürnberg.

Hofstede, G., Neuijen, B., Ohayv, D. D., Sanders, G. 1990. Measuring Organizational Cultures: A Qualitative and Quantitative Study Across Twenty Cases. Administrative Science Quarterly 35 (2): 286-316.

Jasinskaja-Lahti, I., Liebkind, K. 1999. Exploration of the Ethnic Identity among RussianSpeaking Immigrant Adolescents in Finland. Journal of Cross-Cultural Psychology 30 (4): 527-539.

Kalter, F. 2006. Auf der Suche nach einer Erklärung für die spezifischen Arbeitsmarktnachteile von Jugendlichen türkischer Herkunft. Zeitschrift für Soziologie 35: 144-160.

Knight, G. P., Kagan, S. 1977. Acculturation of Prosocial and Competitive Behaviors among Second- and Third-Generation Mexican-American Children. Journal of Cross-Cultural Psychology 8 (3): 273-284.

Liljeberg, H. 2012. Deutsch-Türkische Lebens- und Wertewelten 2012: Ergebnisbericht zu einer repräsentativen Befragung von Türken in Deutschland. Berlin, Antalya. 
Mayring, P. 1993. Einführung in die qualitative Sozialforschung: Eine Anleitung zu qualitativem Denken. Weinheim: Beltz Psychologie-Verl.-Union.

Okamura, J. Y. 1981. Situational ethnicity, in Ethnic and Racial Studies 4 (4): 452-465.

Parrillo, V.N., Donoghue, C. 2005. Updating the Bogardus social distance studies: a new national survey. The Social Science Journal 42: 257-271.

Phinney, J.S. 1992. The Multigroup Ethnic Identity Measure: A New Scale for Use with Diverse Groups. Journal of Adolescent Research 7 (2): 156-176.

Phinney, J. S., Ong, A. D. 2007. Conceptualization and Measurement of Ethnic Identity: Current Status and Future Directions. Journal of Counseling Psychology 54 (3): 271-281.

Portes, A., Zhou, M. 1993. The New Second Generation: Segmented Assimilation and its Variants. The ANNALS of the American Academy of Political and Social Science 530: 74-96.

Pries, L. 1996. Transnationale Soziale Räume: Theoretisch-empirische Skizze am Beispiel der Arbeitswanderungen Mexico - USA. Zeitschrift für Soziologie 25 (6): 456-472.

Rumbaut, R. G. 1994. The Crucible within: Ethnic Identity, Self-Esteem, and Segmented Assimilation among Children of Immigrants. International Migration Review 28 (4): 748-794.

Ryder, A. G., Alden, L. E., Paulhus, D. L. 20oo. Is Acculturation Unidimensional or Bidimensional? A Head-to-Head Comparison in the Prediction of Personality, Self-Identity, and Adjustment. Journal of Personality and Social Psychology 79 (1): 49-65.

Schnell, R. 1990. »Dimensionen ethnischer Identität. In Generation und Identität: Theoretische und empirische Beiträge zur Migrationssoziologie, hrsg. H. Esser, J. Friedrichs, 43-72. Opladen: Westdeutscher Verlag.

Segeritz, M., Walter, O., Stanat, P. 2010. Muster des schulischen Erfolgs von jugendlichen Migranten in Deutschland. Evidenz für segmentierte Assimilation? Kölner Zeitschrift für Soziologie und Sozialpsychologie 62: 113-138.

Singelis, T. M. 1994. The Measurement of Independent and Interdependent Self-Construals. Personality and Social Psychology Bulletin 20 (5): 580-591.

Stryker, S. 1968. Identity Salience and Role Performance: The Relevance of Symbolic Interaction Theory for Family Research. Journal of Marriage and Family 30 (4): 558-564.

Stryker, S., Burke, P. J. 200o. The Past, Present, and Future of an Identity Theory. Social Psychology Quarterly 63 (4): 284-297.

Suinn, R. M. 2009. Acculturation: Measurements and Review of Findings. In Handbook of Mental Health and Acculturation in Asian American Families, hrsg. N.-H. Trinh, 3-24. Heidelberg: Humana Press.

Unger, J. B., Gallaher, P., Shakib, S., Ritt-Olson, A., Palmer, P.H., Johnson, C. A. 2002. The AHIMSA Acculturation Scale: A New Measure of Ac-culturation for Adolescents in a Multicultural Society. In The Journal of Early Adolescence 22 (3): 225-251.

Waters, M. C. 2001. Optional Ethnicities: For Whites Only? In Race, class, and gender: An anthology, hsg. M. L. Andersen, P. H. Collins, 430-439. Belmont (CA): Wadsworth.

Watkins, D., Yau, J., Dahlin, B., Wondimu, H. 1997. The Twenty Statements Test: Some Measurement Issues. Journal of Cross-Cultural Psychology 28 (5): 626-633.

Open Access. This chapter is distributed under the terms of the Creative Commons Attribution Noncommercial License, which permits any noncommercial use, distribution, and reproduction in any medium, provided the original author(s) and source are credited. 